PROCEEDINGS OF THE

AMERICAN MATHEMATICAL SOCIETY

Volume 137, Number 6, June 2009, Pages 2093-2097

S 0002-9939(09)09792-5

Article electronically published on January 29, 2009

\title{
AREA GROWTH RATE OF THE LEVEL SURFACE OF THE POTENTIAL FUNCTION ON THE 3-DIMENSIONAL STEADY GRADIENT RICCI SOLITON
}

\author{
HONGXIN GUO
}

(Communicated by Richard A. Wentworth)

\begin{abstract}
In this short note we show that on a 3-dimensional steady gradient Ricci soliton with positive curvature and which is $\kappa$-noncollapsed on all scales, the scalar curvature and the mean curvature of the level surface of the potential function both decay linearly. Consequently we prove that the area of the level surface grows linearly.
\end{abstract}

\section{INTRODUCTION AND PRELIMINARIES}

In Ricci flow, the Ricci solitons, which are the self-similar solutions evolving only by scalings and diffeomorphisms, have been extensively studied. These special solutions motivate the general analysis of the Ricci flow through monotonicity formulas and their applications. See Hamilton's important work [5]. Recently, quite a few results on the classification and rigidity of the gradient solitons have appeared; see 6, 7, 8, 9, 12, and 13, for example.

In this note we assume $\left(\mathcal{M}^{3}, g, f\right)$ is a steady gradient Ricci soliton which satisfies the equation

$$
R_{a b}+\nabla_{a} \nabla_{b} f=0,1 \leq a, b \leq 3,
$$

where $f$ is called the potential function. Throughout this paper we assume $\mathcal{M}^{3}$ has strictly positive sectional curvature and is $\kappa$-noncollapsed on all scales.

The following claim is due to Perelman (see Remark 11.9 of [10]).

Claim 1.1. I can prove uniqueness in the class of gradient steady solitons.

Related to Perelman's claim, there is a paper 3 by Sun-Chin Chu. He shows that towards spatial infinity, the geometry of a steady soliton becomes more and more rotationally symmetric. Unfortunately, Lemma 2.4 of 3 does not hold because of a mistake in the proof (4]), which affects Theorem 2.3 of [3] and some consequences. In this note, we prove Theorem 2.3 avoiding using Lemma 2.4. Along the way we will also prove that both the scalar curvature and the mean curvature of the level surface of $f$ decay linearly.

Since the scalar curvature $R$ is positive and $R(x) \rightarrow 0$ if $x \rightarrow \infty$ (see Theorem 3.5 of [3]), there is some point $O$, called the origin, where $R$ assumes its maximum.

Received by the editors May 30, 2008, and, in revised form, September 26, 2008.

2000 Mathematics Subject Classification. Primary 53C44.

(C)2009 American Mathematical Society Reverts to public domain 28 years from publication 
Since $\nabla R=2 \operatorname{Rc}(\nabla f, \cdot)$ we have

$$
2 \operatorname{Rc}(\nabla f, \nabla f)(O)=\langle\nabla R(O), \nabla f(O)\rangle=0 .
$$

Then by the strict positivity of Ricci curvature we get $\nabla f(O)=0$. Since $\nabla \nabla f<0$ we know $O$ is the unique maximum point of $f$. Without loss of generality we assume $R(O)=1$ and $f(O)=0$. Since on a steady soliton $R+|\nabla f|^{2}=C$, evaluating $C$ at the origin we have $R+|\nabla f|^{2}=1$. It is obvious that $\lim _{x \rightarrow \infty}|\nabla f|=1$. We have seen from [3] that $-f$ grows linearly. Precisely, for any $0<\delta<1$ there is a compact set outside of which we have

$$
\delta r(x)<-f(x)<r(x),
$$

where $r(x) \doteqdot d(x, O)$

Let $\Sigma(\sigma) \doteqdot\{x \mid f(x)=\sigma\}, \nu \doteqdot-\frac{\nabla f}{|\nabla f|}$, and $\mathcal{R}(\sigma) \doteqdot\{x \mid \sigma \leq f(x) \leq 0\}$. Then $\Sigma$ is a level surface of $f$ and $\nu$ is the unit outward normal vector. The second fundamental form $h$ of $\Sigma$ is defined by $h(X, Y) \doteqdot\left\langle\nabla_{X} \nu, Y\right\rangle$, for any $X, Y \in T(\Sigma)$. The mean curvature $H(\sigma)$ is the trace of $h$. Plugging in the formula for $\nu$, we get

$$
h=-\frac{\nabla \nabla f}{|\nabla f|}=\frac{\mathrm{Rc}}{|\nabla f|} .
$$

Now we present a preliminary lemma which will be used in the next section.

Lemma 1.2. For any $\delta>0$, there is a compact set outside of which

$$
\Delta R+\delta|\mathrm{Rc}|^{2} \geq 0 .
$$

Proof. Argument by contradiction. Suppose there is a sequence of points $\left\{x_{i}\right\}$ with $x_{i} \rightarrow \infty$ such that

$$
\Delta R+\delta|\mathrm{Rc}|^{2} \leq 0
$$

By Theorem 9.66 of [2] we know that

$$
\left(\mathcal{M}^{3}, R_{i} g\left(R_{i}^{-1} t\right), x_{i}\right)
$$

is a limit in the Cheeger-Gromov sense to a solution of the standard shrinking cylinder $S^{2} \times \mathbb{R}$ by passing to a subsequence. On this cylinder, $\Delta_{\infty} R_{\infty}=0$, and $\left|R c_{\infty}\right|>0$, and we get a contradiction.

In inequality (1.2), in particular by choosing $\delta=1$, we have

$$
\langle\nabla R, \nabla f\rangle=\frac{\partial R}{\partial t}=\Delta R+2|\mathrm{Rc}|^{2} \geq|\mathrm{Rc}|^{2} \geq \frac{1}{3} R^{2} .
$$

\section{MAIN RESUlts}

In this section we first show that the scalar curvature $R$ decays linearly. Below we use $C$ and $C_{i}$ to denote constants, and they may vary from line to line. We have

Theorem 2.1 ( $R$ decays linearly). For $r(x)$ large enough, there are positive constants $C_{1}, C_{2}$ such that

$$
\frac{C_{1}}{r(x)} \leq R(x) \leq \frac{C_{2}}{r(x)}
$$


Proof. By Perelman's derivative estimate we have $|\nabla R| \leq C R^{2}$ (see inequality (1.3) in [11]). Apply this along a normal geodesic $\gamma$ emanating from $O$. Then $\langle\nabla R, \dot{\gamma}\rangle \geq-C R^{2}$, so that $\dot{\gamma}\left(\frac{1}{R}\right) \leq C$. Thus

$$
\frac{1}{R(\gamma(r))} \leq \frac{1}{R(\gamma(0))}+C r=1+C r
$$

so that

$$
R(\gamma(r)) \geq \frac{1}{1+C r} .
$$

For $r$ large enough, by choosing an appropriate constant $C_{1}$ we can rewrite the above as

$$
R(x) \geq \frac{C_{1}}{r} .
$$

On the other hand, by inequality (1.3), we have for $r(x)$ large enough that

$$
\langle\nabla f, \nabla R\rangle \geq \frac{1}{3} R^{2}
$$

Let $\beta(\bar{\sigma})$ be the integral curve of $-\frac{\nabla f}{|\nabla f|^{2}}$. Since $|\nabla f| \rightarrow 1$ as $x \rightarrow \infty$, we have outside of a compact set $\mathcal{U}$

$$
\frac{d R(\beta(\bar{\sigma}))}{d \bar{\sigma}}=-\frac{1}{|\nabla f|^{2}}\langle\nabla f, \nabla R\rangle \leq-\frac{1}{2}\langle\nabla f, \nabla R\rangle \leq-\frac{1}{6} R^{2} .
$$

From this differential inequality we get an upper estimate of $R(\beta(\bar{\sigma}))$ in terms of $\bar{\sigma}$, of the order of $1 / \bar{\sigma}$. Since $\bar{\sigma}$ indeed takes the value of $-f$ and is comparable with the distance $r$, we can get a same kind of upper bound of $R(x)$ in terms of $r$. With this intuition in mind, below we give a rigorous argument.

Let $\mathcal{S}_{0}$ be a large sphere outside of $\mathcal{U}$. For any $x$, there is a maximal integral curve $\beta$ passing through $x$ with $\beta(\bar{\sigma})=x$. Let $x_{0}$ be a point of intersection of $\beta$ and $\mathcal{S}_{0}$ and assume $\beta\left(\bar{\sigma}_{0}\right)=x_{0}$. From inequality (2.2) we have

$$
\frac{1}{R(x)}-\frac{1}{R\left(x_{0}\right)} \geq \frac{\bar{\sigma}-\bar{\sigma}_{0}}{6} \text {. }
$$

Let $c_{0} \doteqdot \max \left\{R(\bar{x}): \bar{x} \in \mathcal{S}_{0}\right\}$. Then

$$
\frac{1}{R(x)} \geq \frac{\bar{\sigma}-\bar{\sigma}_{0}}{6}+\frac{1}{c_{0}}
$$

Keep in mind that $\beta$ is an integral curve of $-\frac{\nabla f}{|\nabla f|^{2}}$. We have

$$
-f(x)-\left(-f\left(x_{0}\right)\right)=\int_{\bar{\sigma}_{0}}^{\bar{\sigma}} \frac{d}{d \eta}(-f(\beta(\eta))) d \eta=\int_{\bar{\sigma}_{0}}^{\bar{\sigma}} 1 d \eta=\bar{\sigma}-\bar{\sigma}_{0} .
$$

Let $c_{1} \doteqdot \max \left\{-f(\bar{x}): \bar{x} \in \mathcal{S}_{0}\right\}$. Then

$$
\bar{\sigma}-\bar{\sigma}_{0} \geq-f(x)-c_{1} .
$$

Combining inequalities (2.3) and (2.4), we get

$$
\frac{1}{R(x)} \geq \frac{-f(x)}{6}-\frac{c_{1}}{6}+\frac{1}{c_{0}} .
$$

Since $-f$ grows linearly, there exist constants $c>0$ and $c^{\prime} \doteqdot-c_{1} / 6+1 / c_{0}$ so that

$$
\frac{1}{R(x)} \geq \operatorname{cr}(x)+c^{\prime}
$$


For $r$ large enough, we can choose an appropriate constant $C_{2}>0$ such that

$$
R(x) \leq \frac{C_{2}}{r(x)}
$$

For simplicity, below we will use $H$ to denote $H(\sigma)$.

Corollary 2.2 ( $H$ decays linearly). There are positive constants $C_{1}, C_{2}$ such that when $r(x)$ is large enough we have

$$
\frac{C_{1}}{r(x)} \leq H \leq \frac{C_{2}}{r(x)}
$$

Proof. We have

$$
\operatorname{Rc}(\nu, \nu)=\frac{1}{|\nabla f|^{2}} \operatorname{Rc}(\nabla f, \nabla f)=\frac{1}{2|\nabla f|^{2}}\langle\nabla R, \nabla f\rangle
$$

and

$$
|\langle\nabla R, \nabla f\rangle| \leq|\nabla R| \leq C R^{2} \leq \frac{C}{r^{2}} .
$$

Since $|\nabla f| \rightarrow 1$ we have when $r$ is large enough that

$$
|\operatorname{Rc}(\nu, \nu)| \leq \frac{C}{r^{2}}
$$

Now, by $H=\frac{R-\operatorname{Rc}(\nu, \nu)}{|\nabla f|}$ and inequality (2.1) we get (2.5).

Since $-f$ grows linearly in terms of $r$, immediately we have

Corollary 2.3. There is a compact set outside of which we have

$$
\frac{C_{1}}{-\sigma} \leq H \leq \frac{C_{2}}{-\sigma}
$$

Let $\mathcal{A}_{\sigma} \doteqdot$ area of $\Sigma(\sigma)$ and $\mathcal{V}_{\sigma} \doteqdot$ volume of $\mathcal{R}(\sigma)$. We have

Theorem 2.4. There are constants $C_{1}, C_{2}$ and a compact set outside of which we have

(1) $\mathcal{A}_{\sigma}$ grows linearly: $C_{1} \cdot(-\sigma) \leq \mathcal{A}_{\sigma} \leq C_{2} \cdot(-\sigma)$,

(2) $\mathcal{V}_{\sigma}$ grows quadratically: $C_{1} \sigma^{2} \leq \mathcal{V}_{\sigma} \leq C_{2} \sigma^{2}$.

Proof. The flow equation

$$
\frac{\partial X}{\partial \sigma}=-\frac{1}{|\nabla f|} \nu
$$

evolves the level surfaces into themselves. The area $\mathcal{A}_{\sigma}$ satisfies

$$
\frac{d \mathcal{A}_{\sigma}}{d \sigma}=-\int_{\Sigma} \frac{H}{|\nabla f|} d \mu
$$

Suppose $x$ is far enough from $O$. By inequalities (2.6) and (2.7) we have

$$
\frac{C_{2} \mathcal{A}_{\sigma}}{\sigma} \leq \frac{d \mathcal{A}_{\sigma}}{d \sigma} \leq \frac{C_{1} \mathcal{A}_{\sigma}}{\sigma}
$$

Notice that $\sigma$ is negative, and it is not hard to see that $C_{1} \cdot(-\sigma) \leq \mathcal{A}_{\sigma} \leq C_{2} \cdot(-\sigma)$.

The growth rate of $\mathcal{V}_{\sigma}$ follows from the first part and the co-area formula

$$
\frac{d \mathcal{V}_{\sigma}}{d \sigma}=-\int_{\Sigma} \frac{1}{|\nabla f|} d \mu
$$


Remark 2.5. Theorem 2.4 is Theorem 2.3 in $[3$ with a corrected proof.

\section{ACKNOWLEDGMENTS}

This paper was finished during my Ph.D. studies at the University of California, San Diego. I would like to express my deep gratitude to my advisor and friend, Bennett Chow, whose guidance and support were crucial to my mathematical research. I would like to thank Professors Sun-Chin Chu, Peng Lu and Lei Ni for their helpful discussions and suggestions. I would also like to thank the referee for helpful suggestions.

\section{REFERENCES}

1. Chow, Bennett; Chu, Sun-Chin; Glickenstein, David; Guenther, Christine; Isenberg, James; Ivey, Tom; Knopf, Dan; Lu, Peng; Luo, Feng; Ni, Lei. The Ricci flow: Techniques and applications, Part I: Geometric aspects. Math. Surveys Monogr. 135, Amer. Math. Soc., Providence, RI, 2007. MR2302600 (2008f:53088)

2. Chow, Bennett; Lu, Peng; Ni, Lei. Hamilton's Ricci flow. Grad. Studies in Math. 77, Amer. Math. Soc., Providence, RI; and Science Press, New York, 2006. MR.2274812 (2008a:53068)

3. Chu, Sun-Chin. Geometry of 3-dimensional gradient Ricci solitons with positive curvature. Comm. Anal. Geom. 13 (2005), no. 1, 129-150. MR2154669 (2006h:53060)

4. Chu, Sun-Chin. Personal communications.

5. Hamilton, Richard S. The formation of singularities in the Ricci flow. Surveys in Differential Geometry, Vol. II (Cambridge, MA, 1993), 7-136, Internat. Press, Cambridge, MA, 1995. MR.1375255 (97e:53075)

6. Lott, John. Dimensional reduction and the long-time behavior of Ricci flow. arXiv: 0711.4063.

7. Naber, Aaron. Noncompact shrinking 4-solitons with nonnegative curvature. arXiv: 0710.5579 .

8. Ni, Lei; Wallach, Nolan. On a classification of the gradient shrinking solitons. Math. Res. Lett. 15 (2008), no. 5, 941-955.

9. Ni, Lei; Wallach, Nolan. On 4-dimensional gradient shrinking solitons. Int. Math. Res. Notices (2008), vol. 2008, article ID rnm152.

10. Perelman, Grisha. The entropy formula for the Ricci flow and its geometric applications. arXiv:math.DG/0211159.

11. Perelman, Grisha. Ricci flow with surgery on three-manifolds. arXiv:math.DG/ 0303109.

12. Petersen, Peter; Wylie, William. Rigidity of gradient Ricci solitons. arXiv:0710.3174.

13. Petersen, Peter; Wylie, William. On the classification of gradient Ricci solitons. arXiv:0712.1298.

School of Mathematics and Information Science, Wenzhou University, Wenzhou, Zhejiang, 325035 People's Republic of China

E-mail address: hguo2006@gmail.com 